\title{
Environmental Considerations in Thermodynamic Investigation of Bulk Samples by SEM
}

\author{
M.J.Castagna, ${ }^{*}$, Hiroshi Fujitani, ${ }^{* *}$ and D.W.Phifer, ${ }^{* * *}$
}

* FEI Company, North American Nanoport, 5350 NE Dawson Creek Dr, Hillsboro, OR 97124-5793

** FEI Company Japan Ltd., Nanoport Japan, NSS- II Bldg.1F 13-34 Konan 2-chome, Minato-ku, Tokyo 108-0075 Japan.

*** FEI Company Netherlands, Building AAE, Achtseweg Noord 5, Eindhoven 5600 KA, The Netherlands.

Utilizing a modern field emission environmental scanning electron microscope (FE-ESEM) coupled with integrated heating and cooling stages, it is possible to observe materials in-situ using various experimental conditions. The FE-ESEM yields high contrast, high resolution imaging[1] in different gaseous environments, allowing for comparative experimentation while viewing the results directly at the surface. With a hot stage and environmental gas control integrated into the FE-ESEM's user interface, grain boundary growth, void generation, sintering[2], surface reorganization, and the melting of various metals has been observed in real time and time compressed movies are generated to record these results. These reactions can be altered by the atmosphere in the FE-ESEM and results are readily observable in-situ[3] throughout the whole experimental temperature range using gasamplified signal amplification which is insensitive to light or heat. Results of some experiments illustrate some opportunities and limitations of bulk material research inside an environmental SEM. For example, oxidizing reactions are easily favored even in inert environments, however, useful slowing of these reactions can be observed. The ability to observe changes in material transformation with various temperature-atmosphere has implications for energy research, metals research and semiconductor materials.

References

[1] M. Schmied, et al., Microsc Microanal 11(Suppl 2), (2005) 412.

[2] Srinivasan N. Iyengar., PowderMet Meeting (2009) 2009-01-0061.

[3] T. Jonsson, et al., Corrosion Science Volume 51, Issue 9, (2009) 1914. 


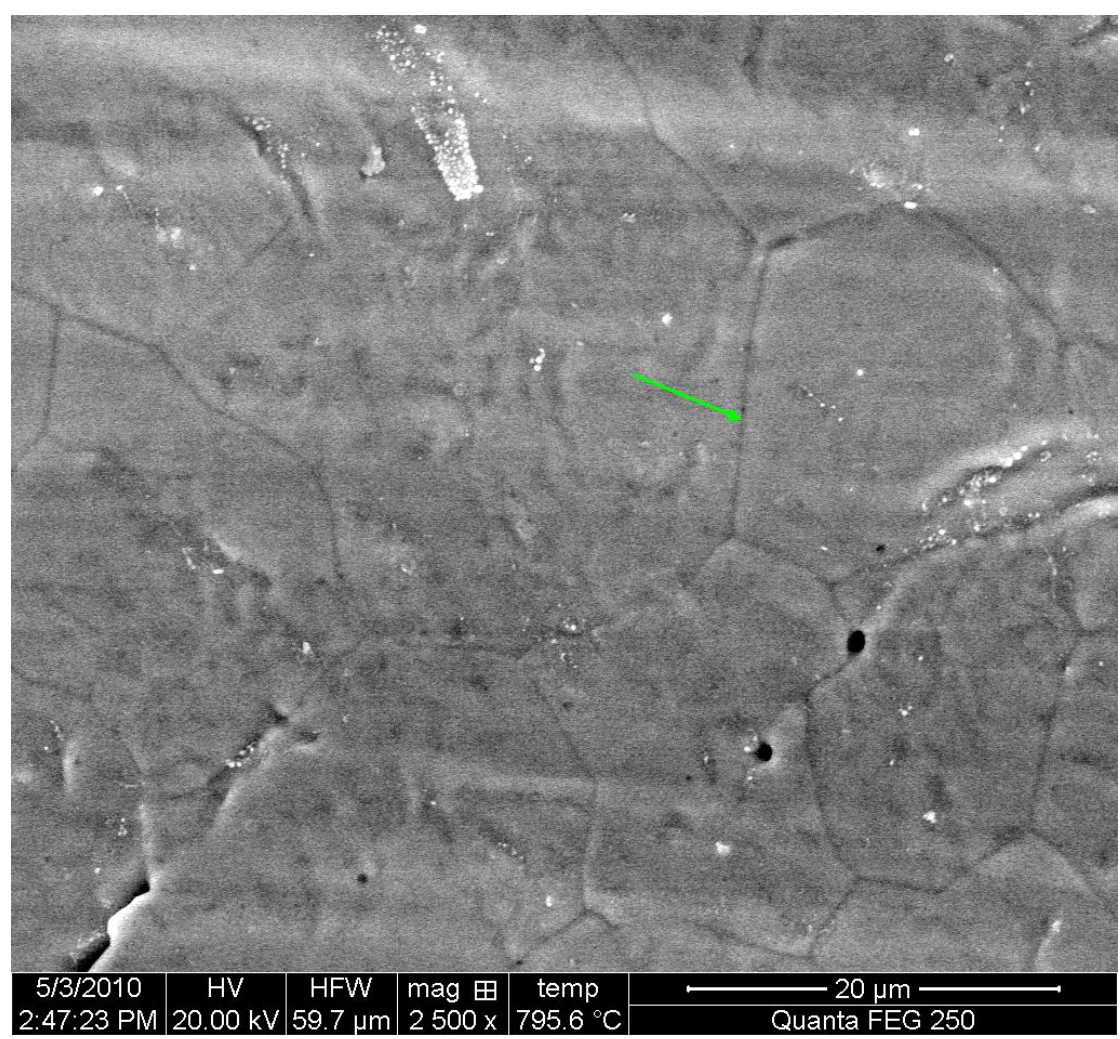

FIG. 1. Image of copper surface at $795.6^{\circ} \mathrm{C}$ prior to grain boundry shifting. The arrow indicates a boundry which will be shown to migrate.

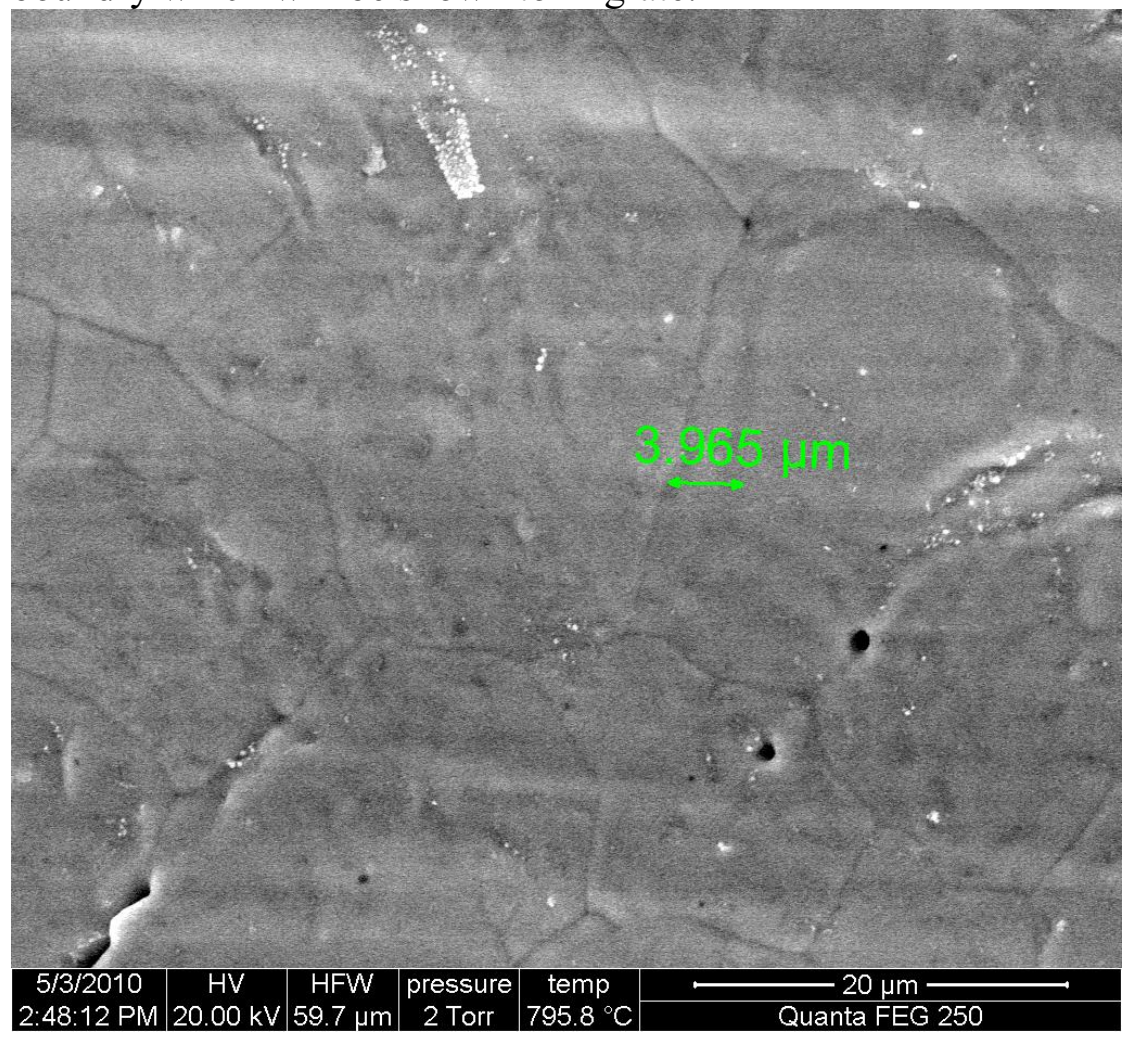

FIG. 2. Image of copper surface $<1$ minute after image in figure 1. Note: Grain boundry has shifted $\sim 4 \mu \mathrm{m}$ from location in figure 1 . 